\title{
Estimation de la diversité parmi les souches de la collection CNRZ : application de la RAPD à un groupe de lactobacilles
}

\author{
P Tailliez 1, P Quénée 1, A Chopin 2 \\ 1 Unité de recherches laitières, Collection CNRZ; \\ ${ }^{2}$ Laboratoire de génétique microbienne, Inra, 78352 Jouy-en-Josas cedex, France
}

\begin{abstract}
Résumé - Le rôle principal de la collection CNRZ de bactéries lactiques a été jusqu'à présent de conserver les souches qui y sont déposées. Outre les tests classiques nécessaires à l'identification, la caractérisation de ces dernières reste sommaire. De plus, la taille de la collection rend hors de portée l'étude systématique des souches qui la composent. II est donc nécessaire de limiter la caractérisation plus détaillée à un nombre restreint d'individus, représentatifs de l'ensemble. C'est pourquoi nous avons cherché à mesurer la diversité qui existe entre les souches. La technique utilisée est la RAPD (randomly amplified polymorphic DNA fingerprinting) qui permet une comparaison directe des séquences d'ADN du génome des souches. Des régions du génome sont amplifiées de manière aléatoire par PCR (DNA polymerase chain reaction), en utilisant des amorces d'ADN composées d'une séquence arbitraire de dix nucléotides. Les bandes obtenues sont ensuite révélées par électrophorèse. Les similitudes de profils traduisent des similarités du génome. Cette approche a été appliquée à un ensemble de 78 souches de lactobacilles et a révélé quatre groupes de profils RAPD. Les groupes G1 à G3 renferment des souches de $L b$ pentosus tandis que $G 4$ renferme des souches de $L b$ plantarum. Neuf souches n'appartiennent pas à ces groupes, et pourraient représenter des types rares ou sous-représentés dans la collection. Cinquante-neuf souches présentent un profil identique à celui d'au moins une autre souche. Parmi elles, 39 ont été isolées simultanément du même produit et sont probablement des doublons. Enfin, parmi 18 souches dont le statut taxonomique était incertain, 11 ont pu être identifiées comme Lb pentosus ou Lb plantarum.
\end{abstract}

\section{RAPD / diversité / lactobacille / taxonomie / collection}

Summary - Estimation of the diversity among the strains of the CNRZ collection: application of the RAPD technique to a group of lactobacilli. The main function of the CNRZ collection of dairy bacteria has been the preservation of the deposited strains. Up to now, strains have been mostly studied for identification purposes. Given the high number of strains in the collection, a detailed study of all of them is out of reach. It is thus necessary to concentrate the studies on a limited number of representative strains. Toward the identification of these representative strains, we have undertaken to characterize by RAPD (randomly amplified polymorphic DNA fingerprinting) the genome diversity which exists between the strains in the collection. In this technique, arbitrarily chosen DNA regions are amplified by PCR (DNA polymerase chain reaction) using 10 nucleotide-long-primers. Amplified bands are visualized by electrophoresis. Similar profiles indicate similar genomes. This approach has been applied to a group of 78 lactobacilli strains and revealed 4 RAPD groups. Groups G1 to G3 contain strains of $\mathrm{Lb}$ pentosus when group $G 4$ contains strains of $\mathrm{Lb}$ plantarum. Nine strains did not belong to any of these groups and may belong to rare or under-represented types of strains. Fifty-nine strains had a pro- 
file identical to the one of at least one other strain. Among them, 39 were isolated from identical origin and at the same time, and most probably correspond to multiple isolates of the same strains. Finally, among 18 strains of uncertain taxonomic status, 11 were identified by their RAPD profile as belonging to the Lb pentosus or Lb plantarum species.

RAPD / diversity / lactobacillus / taxonomy / collection

\section{INTRODUCTION}

Le rôle principal des collections de microorganismes a été jusqu'à présent de conserver les souches qui y sont déposées. En général, à part les tests classiques nécessaires à l'identification, la caractérisation des souches reste sommaire. Cela est dû au fait que les collections rassemblent de grands nombres de souches et que les caractères potentiellement intéressants sont très nombreux et souvent difficiles à mesurer. C'est en particulier le cas de la collection du Centre national de recherches zootechniques (CNRZ), qui conserve et met à la disposition des chercheurs et des industriels plus d'un millier de souches de bactéries lactiques. Outre le nom d'espèce, qui est généralement connu, peu d'informations sont disponibles sur les caractéristiques des souches. De plus, la taille de la collection rend hors de portée l'étude systématique des souches qui la composent. II est donc nécessaire de limiter la caractérisation plus détaillée à un nombre restreint d'individus représentatifs de l'ensemble. Cela implique de pouvoir mesurer la ressemblance et la diversité qui existent entre les souches de la collection de façon à repérer et à faire l'inventaire de celles qui sont réellement différentes.

\section{COMMENT MESURER LA DIVERSITÉ ?}

Les procédés classiques de caractérisation des souches bactériennes tels que les caractérisations biochimiques ou nutritionnelles, l'analyse des protéines totales ou de la composition de la paroi ou de la membrane, les réactions immunologiques ou la détermination du contenu plasmidique ne permettent pas, en général, de distinguer des souches apparentées de la même espèce (Le Bourgeois et al, 1993).

Les méthodes permettant de mesurer la diversité existant entre souches de microorganismes apparentées sont basées sur la comparaison de la séquence de leurs génomes. Par exemple, les comparaisons de séquence d'ARN ribosomal 165 et les mesures d'homologie ADN/ADN peuvent être utilisées pour rattacher une souche inconnue à un genre et/ou à une espèce. II s'agit cependant d'analyses lourdes qui ne permettent pas d'observer facilement la diversité au sein d'une espèce. Deux méthodes semblent suffisamment rapides pour pouvoir analyser de nombreuses souches et sont susceptibles de révéler des différences entre souches d'une même espèce. II s'agit de l'électrophorèse en champs pulsés de grands fragments d'ADN (Le Bourgeois et al, 1993) et de la RAPD (Welsh et McClelland, 1990 ; Williams et al, 1990). Dans la première méthode, l'ADN total de la souche est digéré par une enzyme de restriction reconnaissant des sites rares. Cette digestion génère un nombre limité de fragments chromosomiques qui sont ensuite révélés par électrophorèse en champs pulsés (PFGE : pulsed field gel electrophoresis). En RAPD (randomly amplified polymorphic DNA), des séquences du génome sont synthétisées par la technique de l'amplification en chaîne de l'ADN par la polymérase Taq (PCR : polymerase chain reaction) en utilisant, dans des conditions de faible stringence, de courtes amorces d'ADN de séquence nucléotidique choisie de manière aléatoire. Le principe de cette méthode est détaillé sur la figure 1. Des 
souches identiques produiront des profils similaires et des souches différentes produiront des profils variés. Cette dernière méthode offre l'avantage d'avoir une résolution qui peut être augmentée à volonté en augmentant le nombre d'amorces utilisées. Cette possibilité nous a conduit à préférer cette méthode pour évaluer la diversité parmi les souches de la collection CNRZ.

\section{L'EXEMPLE DE LACTOBACILLUS PLANTARUM ET DE Lb PENTOSUS}

Nous présentons ici la caractérisation d'un ensemble de souches de $L b$ plantarum et de $L b$ pentosus (tableau I). Ce groupe a été choisi car il rassemble des souches d'origines extrêmement variées, donc susceptibles d'être très différentes les unes des autres. En outre, les souches appartenant à ces deux espèces posent des problèmes d'identification. En effet, il a été montré par mesure d'homologie ADN/ADN que l'espèce $L b$ plantarum regroupait en fait deux espèces : Lb plantarum et $L b$ pentosus (Dellaglio et al, 1975 ; Zanoni et al, 1987). II a été proposé que les souches de cette dernière espèce soient différenciées par leur aptitude à produire de l'acide à partir du glycérol et du D(-)xylose (Zanoni et al, 1987). D'après les résultats obtenus avec les souches de notre collection (tableau I), il semble que la production d'acide à partir de mélézitose puisse également être utilisée comme critère taxonomique. Les souches de Lb plantarum seraient alors xylose-, glycérol-et mélézitose+ et les souches de $L b$ pentosus seraient xylose+, glycérol+ et mélézitose-. Cependant, l'identification par ces caractères phénotypiques n'est pas satisfaisante. Parmi 17 souches de lactobacilles examinées par Zanoni et al (1987), certaines présentaient des phénotypes atypiques. De même, dans notre collection, parmi 78 souches étudiées, 18 présentent des phénotypes intermédiaires et ne peuvent donc être identifiées par ces critères. Elles sont désignées $L b$ species (tableau I).

\section{CONDUITE DE LA RAPD}

L'analyse de ces souches par RAPD commence par la purification de leur ADN en utilisant une méthode décrite précédemment (de los Reyes-Gavilan et al, 1992). Après contrôle de la quantité et de la qualité de cet ADN par électrophorèse, la RAPD est réalisée dans $100 \mu \mathrm{l}$ de tampon Tris $\mathrm{HCl}$, $10 \mathrm{mmol} / \mathrm{l}\left(\mathrm{pH} 9,0\right.$ à $\left.25^{\circ} \mathrm{C}\right)$ contenant 20 à $100 \mathrm{ng}$ d'ADN, $1,5 \mathrm{mmol} / \mathrm{l}$ de $\mathrm{MgCl}_{2}$, $0,5 \mu \mathrm{mol} / /$ d'amorce (Bioprobe), 2,5 unités de polymérase (AmpliTaq, Appligène) et $200 \mu \mathrm{mol} / \mathrm{l}$ de chacun des nucléotides dATP, dCTP, dGTP et dTTP (Boehringer Mannheim). Deux amorces sont utilisées séquentiellement dans deux réactions PCR différentes : 5'TGCTCTGCCC (B06) et 5'GTCCACACGG (B08). Ces réactions sont réalisées dans l'appareil Perkin Elmer, modèle 9600 et comportent 30 cycles d'amplification $\left(94^{\circ} \mathrm{C}\right.$ pendant $1 \mathrm{~min}, 36^{\circ} \mathrm{C}$ pendant $2 \mathrm{~min}, 72^{\circ} \mathrm{C}$ pendant $2 \mathrm{~min}$ ). Après amplification, $20 \mu \mathrm{l}$ de produits PCR sont soumis à l'électrophorèse en agarose SEAKEM GTG $1 \%$ (Tebu). Les produits d'amplification sont détectés après coloration du gel dans une solution de bromure d'éthidium. Un marqueur de taille (polymère de $123 \mathrm{pb}$, Gibco BRL) est inclus dans chaque gel.

Pour regrouper les souches en fonction de leurs profils RAPD, deux approches sont suivies habituellement. Dans le cas où les profils appartiennent clairement à des classes distinctes, leur comparaison visuelle et leur classification sont aisées (Kersulyte et al, 1992 ; Brikun et al, 1994). En revanche, quand l'existence de classes n'est pas évidente ou que des profils intermédiaires entre les classes existent, il est nécessaire de quantifier la ressemblance entre paires de profils. Cela peut être fait en notant la présence ou l'absence de bandes 


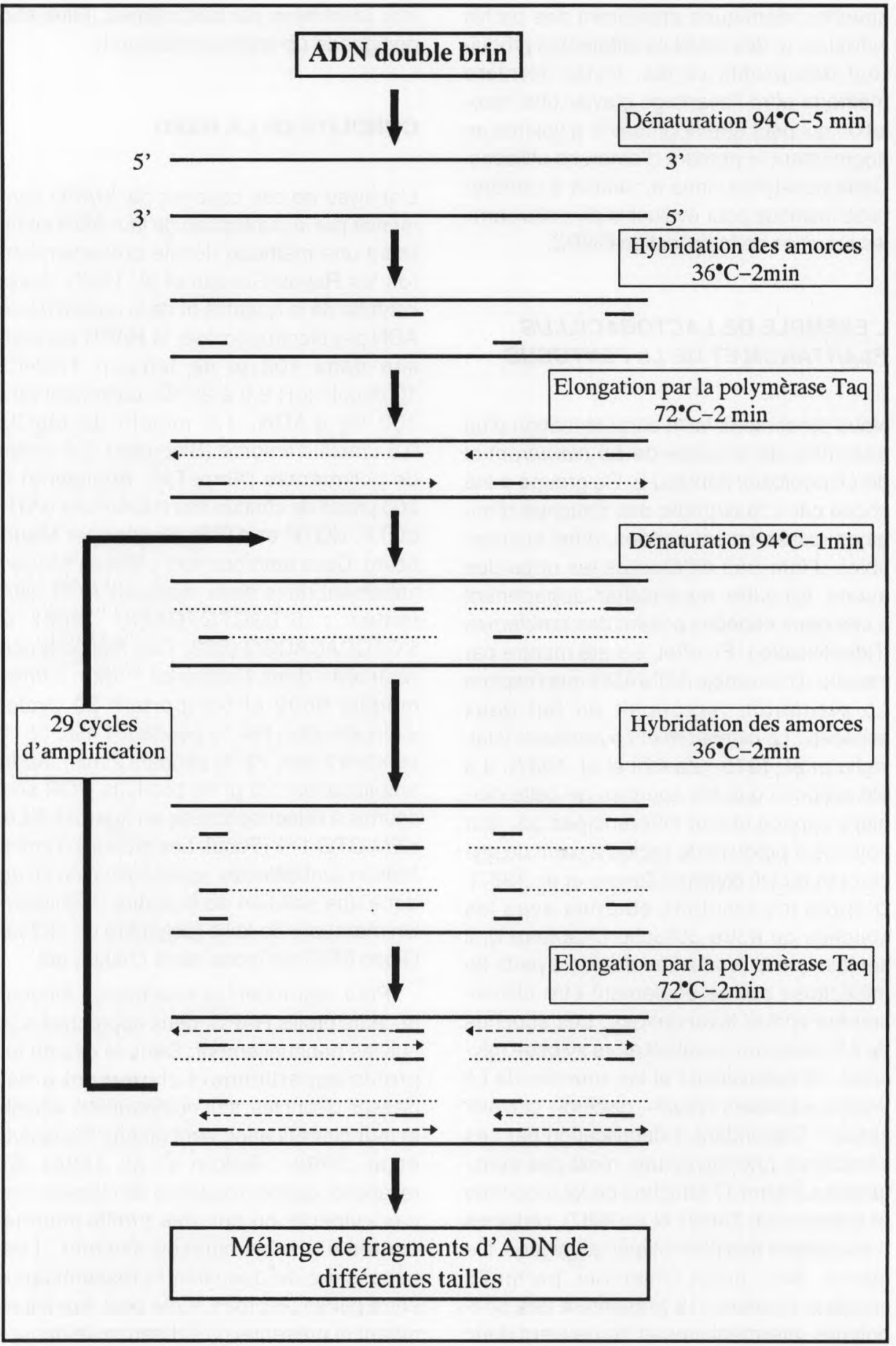


spécifiques (Welsh et al, 1992) ou en calculant le rapport entre le nombre de bandes communes aux deux pistes et la somme des bandes présentes dans les deux pistes pour en déduire un coefficient de similarité (Fekete et al, 1992). Dans notre cas cependant, ce type d'interprétation était rendu difficile par le grand nombre de souches examinées, le nombre élevé de bandes présentes dans les pistes, et surtout les différences d'intensité entre les bandes. II était donc nécessaire de prendre en compte dans l'interprétation, d'une façon objective et automatique, non seulement les positions des bandes mais aussi leurs intensités respectives. Pour cela, les gels sont photographiés et les négatifs Polaroïd numérisés par un scanner (Hewlet Packard ScanJet IIcx/T). L'image est ensuite traitée en utilisant le logiciel GelCompar (Applied Maths, Belgique). Ce dernier permet de normaliser les profils afin de minimiser les différences de migration entre gels, de calculer les coefficients de símilarité de Pearson (Sneath et Sokal, 1973) entre profils en tenant compte de la position et de l'intensité des différents produits RAPD, et de regrouper ces profils à partir de ces coefficients, en utilisant la méthode d'agrégation hiérarchique UPGMA (unweighted pair group method using arithmetic averages) (Sokal et Michener, 1958). Enfin, le programme est capable de générer une banque de profils permettant l'identification et la classification de toute nouvelle souche (Vauterin et Vauterin, 1992).

\section{RÉSULTATS}

Cette technique a été appliquée aux 78 souches de lactobacilles du groupe $L b$ plantarum- $L b$ pentosus de notre collection en utilisant les deux amorces B06 et B08. Les résultats obtenus sont montrés sur la figure 2 .

\section{Mise en évidence des souches similaires}

L'examen des profils RAPD révèle des groupes de profils identiques ou extrêmement similaires entre eux. Cela se traduit par des coefficients de similarité supérieurs à $80 \%$. Ces valeurs sont de l'ordre de celles que l'on mesure en analysant plusieurs fois une même souche. Cette valeur de $80 \%$ correspond donc au seuil minimum de répétabilité de la méthode et ces groupes se composent de souches que l'analyse RAPD ne parvient pas à distinguer. $Y$ sont rassemblées des souches probablement très proches, voire identiques. En effet, ils contiennent souvent des souches isolées d'un même type de produits, suggérant qu'il peut s'agir en fait d'isolements multiples de la même souche. Sur ces critères, on peut considérer que, parmi les 78 souches de la collection,

Fig 1. La technique RAPD est fondée sur la réaction de polymérisation en chaîne de l'ADN (Ou PCR). Elle permet d'amplifier des régions du génome en utilisant des amorces ou sondes courtes (dix bases) dont les séquences nucléotidiques sont choisies de manière arbitraire. L'ADN cible de la bactérie est d'abord dénaturé (séparation des doubles brins) par chauffage à $94^{\circ} \mathrm{C}$. Puis l'hybridation des sondes sur cet ADN est effectuée en condition peu stringente à $36^{\circ} \mathrm{C}$. Enfin, la synthèse du brin complémentaire par la polymérase Taq s'effectue à $72^{\circ} \mathrm{C}$, température optimale de fonctionnement de l'enzyme. Une trentaine de cycles (dénaturation-hybridation-synthèse) sont nécessaires pour synthétiser des quantités suffisantes des différents fragments RAPD qui sont ensuite visualisés par électrophorèse. ( ) ADN; (-) amorce ; ) ADN néosynthétisé.

The RAPD technique is based upon the polymerase chain reaction (PCR). It allows, using short arbitrary primers, the amplification of randomly chosen regions of the genome. The double stranded bacterial DNA is first denaturated by heating at $94{ }^{\circ} \mathrm{C}$. Hybridization of the primers is done at low stringency $\left(36{ }^{\circ} \mathrm{C}\right)$. The complementary strand is synthesized by the DNA Taq polymerase at $72{ }^{\circ} \mathrm{C}$. After 30 cycles (denaturation-hybridization-synthesis), enough of the different RAPD fragments is produced to allow their detection by gel electrophoresis. ( ) DNA, ( $)$ primer, ( ) synthesized DNA. 
Tableau I. Caractéristiques des souches étudiées.

Characteristics of the strains.






\begin{tabular}{|c|c|c|c|c|c|}
\hline $\begin{array}{l}\text { Nom } \\
\text { d'espèce }\end{array}$ & $\begin{array}{l}\text { Numéro } \\
\text { CNRZ }\end{array}$ & $\begin{array}{c}\text { Autre } \\
\text { numéro }\end{array}$ & $\begin{array}{l}\text { Acide produit à partir de } \\
D \text {-xylose glycérol mélézitose }\end{array}$ & $\begin{array}{l}\text { Groupe } \\
\text { RAPD }\end{array}$ & Origine \\
\hline
\end{tabular}

\begin{tabular}{|c|c|c|c|c|c|c|}
\hline Lb species & 1544 & - & + & + & G3 & Fermentation d'olives espagnoles \\
\hline Lb species & 1545 & - & + & + & G3 & Fermentation d'olives espagnoles \\
\hline Lb pentosus & 1546 & + & + & - & G3 & Fermentation d'olives espagnoles \\
\hline Lb pentosus & 1547 & + & + & - & G1 & Fermentation d'olives espagnoles \\
\hline Lb species & 1548 & - & + & - & i & Fermentation d'olives espagnoles \\
\hline Lb pentosus & 1549 & + & + & - & G2 & Fermentation d'olives espagnoles \\
\hline Lb pentosus & 1550 & + & + & - & G2 & Fermentation d'olives espagnoles \\
\hline Lb pentosus & 1551 & + & + & - & G2 & Fermentation d'olives espagnoles \\
\hline Lb pentosus & 1552 & + & + & - & G3 & Fermentation d'olives espagnoles \\
\hline Lb pentosus & 1553 & + & + & - & G3 & Fermentation d'olives espagnoles \\
\hline Lb pentosus & 1554 & + & + & - & G2 & Fermentation d'olives espagnoles \\
\hline Lb pentosus & 1555 & + & + & - & G1 & Fermentation d'olives espagnoles \\
\hline Lb pentosus & 1556 & + & + & - & G1 & Fermentation d'olives espagnoles \\
\hline Lb pentosus & 1557 & + & + & - & G2 & Fermentation d'olives espagnoles \\
\hline Lb pentosus & 1558 & + & + & - & G3 & Fermentation d'olives espagnoles \\
\hline Lb pentosus & 1559 & + & + & - & G1 & Fermentation d'olives espagnoles \\
\hline Lb pentosus & 1560 & + & + & - & G1 & Fermentation d'olives espagnoles \\
\hline Lb pentosus & 1561 & + & + & - & G3 & Fermentation d'olives espagnoles \\
\hline Lb pentosus & 1562 & + & + & - & G3 & Fermentation d'olives espagnoles \\
\hline Lb pentosus & 1563 & + & + & - & G3 & Fermentation d'olives espagnoles \\
\hline Lb pentosus & 1564 & + & + & - & G1 & Fermentation d'olives espagnoles \\
\hline Lb pentosus & 1565 & + & + & - & G1 & Fermentation d'olives espagnoles \\
\hline Lb pentosus & 1566 & + & + & - & G3 & Fermentation d'olives espagnoles \\
\hline Lb species & 1567 & + & - & - & G1 & Fermentation d'olives espagnoles \\
\hline Lb pentosus & 1568 & + & + & - & G1 & Fermentation d'olives espagnoles \\
\hline Lb pentosus & 1569 & + & + & - & G1 & Fermentation d'olives espagnoles \\
\hline Lb pentosus & 1570 & + & + & - & G1 & Fermentation d'olives espagnoles \\
\hline
\end{tabular}




\begin{tabular}{|c|c|c|c|c|c|c|c|}
\hline \multirow{2}{*}{$\begin{array}{l}\text { Nom } \\
\text { d'espèce }\end{array}$} & \multirow{2}{*}{$\begin{array}{l}\text { Numéro } \\
\text { CNRZ }\end{array}$} & \multirow{2}{*}{$\begin{array}{l}\text { Autre } \\
\text { numéro }\end{array}$} & \multicolumn{3}{|c|}{ Acide produit à partir de } & \multirow{2}{*}{$\begin{array}{l}\text { Groupe } \\
\text { RAPD }\end{array}$} & \multirow[t]{2}{*}{ Origine } \\
\hline & & & $D$-xylose & glycérol & mélézitose & & \\
\hline Lb plantarum & 1571 & ATCC8014 & - & - & + & G4 & \\
\hline Lb plantarum & 1572 & & - & - & + & G4 & Fromage espagnol \\
\hline Lb pentosus & 1573 & & + & + & - & G3 & Fermentation d'olives espagnoles \\
\hline Lb plantarum & 1602 & & - & - & + & G4 & Fromage artisanal yougoslave \\
\hline Lb plantarum & 1603 & & - & - & + & G4 & Fromage artisanal yougoslave \\
\hline Lb plantarum & 1604 & & - & - & + & G4 & Fromage artisanal yougoslave \\
\hline Lb plantarum & 1605 & & - & - & + & G4 & Fromage artisanal yougoslave \\
\hline Lb plantarum & 1606 & & - & - & + & G4 & Fromage artisanal yougoslave \\
\hline Lb plantarum & 1607 & & - & - & + & G4 & Fromage artisanal yougoslave \\
\hline Lb plantarum & 1669 & & - & - & + & G4 & \\
\hline Lb plantarum & 1838 & & - & - & + & G4 & \\
\hline Lb plantarum & 1839 & & - & - & + & G4 & \\
\hline Lb plantarum & 1849 & & - & - & + & i & \\
\hline Lb plantarum & 1850 & & - & - & + & i & \\
\hline Lb plantarum & 1851 & & - & - & + & i & Fromage espagnol \\
\hline Lb pentosus & 1858 & ATCC8041 & + & + & - & G3 & Ensilage de maïs, souche type \\
\hline Lb species & 1859 & TISTR542 & - & - & - & i & Porc fermenté thaïlandais \\
\hline Lb species & 1860 & TISTR543 & - & - & - & $\mathrm{i}$ & Porc fermenté thaïlandais \\
\hline Lb species & 1861 & TISTR845 & + & + & + & $\mathrm{i}$ & Saucisse de porc aigre thaïlandais \\
\hline Lb pentosus & 1862 & TISTR851 & + & + & - & G3 & Poisson fermenté thaïlandais \\
\hline Lb pentosus & 1863 & TISTR853 & + & + & - & G1 & Gâteau de poisson thaïlandais \\
\hline Lb pentosus & 1864 & TISTR859 & + & + & - & G3 & Saumure thaïlandaise \\
\hline Lb species & 1865 & TISTR865 & - & + & - & G3 & Oignons marinés thaïlandais \\
\hline Lb species & 1866 & TISTR869 & + & + . & + & G2 & Bambous fermentés thaïlandais \\
\hline Lb pentosus & 1867 & TISTR871 & + & + & - & G2 & $\begin{array}{l}\text { Feuilles de thé fermentées } \\
\text { thaïlandaises }\end{array}$ \\
\hline
\end{tabular}




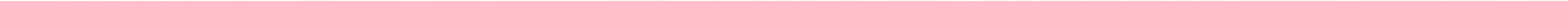


Coefficient de similarité en \%

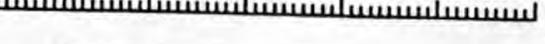

B08

B06
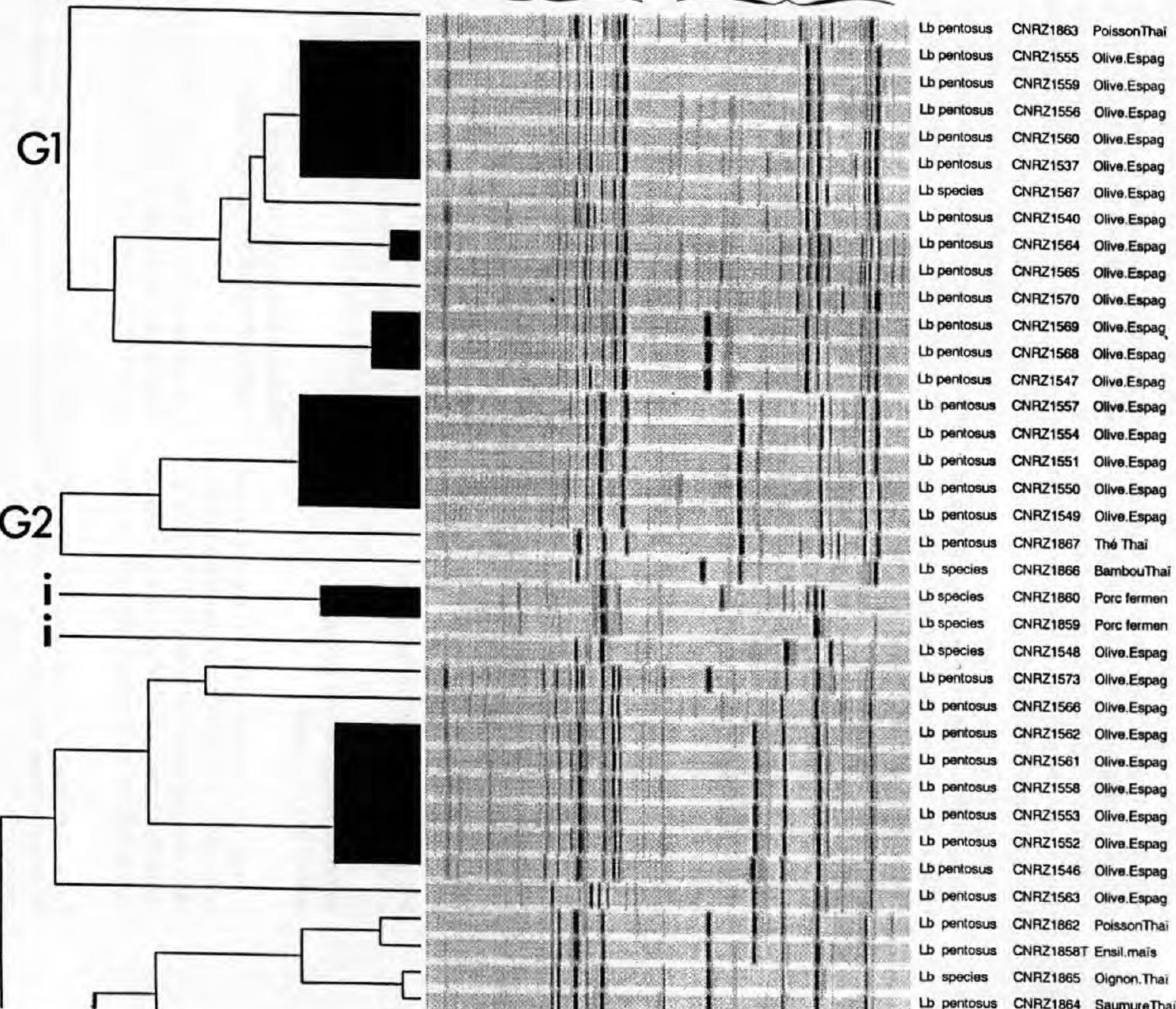
29 sont probablement des doublons, ce qui ramène à 49 le nombre de souches potentiellement différentes dans la collection. Les doublons sont particulièrement fréquents dans le cas des souches isolées de fermentation d'olives vertes d'Espagne.

Cependant, dans plusieurs cas, des souches indifférenciables par l'analyse RAPD ont été isolées de produits fabriqués dans différents pays et à des époques différentes. C'est le cas des souches de $L b$ pentosus CNRZ1862 (isolée de poisson fermenté en Thaïlande) et CNRZ1858 (souche type), isolée d'ensilage de maīs. Dans l'espèce $L b$ plantarum, ces exemples sont plus fréquents et incluent les souches CNRZ1228 (ou CNRZ1229) et CNRZ211 (souche type) isolées respectivement de fromage égyptien et de chou rouge ; CNRZ432 et CNRZ738, isolées respectivement d'un levain de boulangerie et d'ensilage ; CNRZ1220 et CNRZ1310, isolées respectivement de fromages égyptien et espagnol. Cela indique que des clones très proches peuvent être largement disséminés géographiquement tout en s'étant adaptés à des environnements variés.

\section{Mise en évidence de groupes parmi les souches}

L'examen des profils RAPD montre aussi qu'il existe des groupes de souches possédant des profils significativement différents mais renfermant des bandes communes ce qui permet de mesurer valablement un coef- ficient de similarité entre ces souches et de les regrouper. Cependant, ces similarités, qu'utilise la méthode UPGMA pour former des groupes, doivent être interprétées avec précaution. En effet, une bande d'amplification RAPD présente dans deux profils ne peut être considérée comme un indice de proximité taxonomique entre les souches que si elle représente la même séquence génomique. Cela est probable quand les deux profils présentent de nombreuses bandes de même taille en commun. Cela devient beaucoup plus douteux quand les profils ne se ressemblent que par une ou deux bandes de taille voisine. Dans ce cas, on ne peut pas exclure que ces bandes représentent des séquences différentes du génome dont les tailles sont semblables de façon fortuite. II n'est alors pas possible, sur la base de ces seuls résultats de RAPD, de mesurer la parenté existant entre les groupes. Ainsi, seule la partie du regroupement proposée par la méthode UPGMA et qui a pu être confirmée par inspection visuelle des profils, est reportée sur la figure 2. L'analyse révèle alors quatre groupes de souches (indiqués G1 à G4 sur la figure 2) et neuf souches donnant des profils RAPD uniques (notés i pour isolés sur la figure 2).

\section{Identification des souches à statut taxonomique incertain}

Les groupes des souches ainsi définis correspondent remarquablement bien avec leur classement dans les deux espèces. Le

Fig 2. Comparaison et regroupement des profils RAPD obtenus pour 78 souches de lactobacilles hétérofermentaires facultatifs. Pour chaque souche, deux profils ont été obtenus, l'un avec l'amorce B06, l'autre avec B08. Ils ont ensuite été combinés par le logiciel GelCompar et sont regroupés par la méthode UPGMA. Chacun des groupes (G1 à G4) contient des profils ayant un nombre significatif de bandes amplifiées en commun. Les carrés noirs regroupent les souches isolées d'un même type de produits et ayant des profils similaires à plus de $80 \%$. i indique les profils isolés.

Comparison and grouping of 78 RAPD profiles obtained from facultatively heterofermentative lactobacilli. For each strain, two profiles were obtained, one with primer B06, the other one with primer B08. Patterns were combined using the GelCompar software and grouped using the UPGMA method. Each group (G1 to G4) is composed of patterns with many common bands of amplified DNA fragments. Black squares indicate strains which have been isolated from a same kind of product and which have profiles with more than $80 \%$ of similarity, $i$ indicates isolated profiles. 
groupe G4 contient 26 souches dont 23 préalablement identifiées comme $\mathrm{Lb}$ plantarum et trois dont l'espèce était indéterminée. L'analyse RAPD montre que les profils de ces souches (CNRZ1220, CNRZ1229 et $C N R Z 1311$ ) sont très similaires à ceux des souches de Lb plantarum. Nous proposons donc qu'elles soient classées dans cette espèce. De même, les groupes G1 à G3 contiennent des souches préalablement identifiées comme $L b$ pentosus à l'exception de 13 souches dont l'espèce était indéterminée. Pour les mêmes raisons, nous proposons que les huit souches $L b$ species du groupe G3 (contenant la souche type) soient classées dans l'espèce Lb pentosus. Pour ce qui concerne les souches non identifiées et n'appartenant pas à un groupe RAPD, nos résultats ne permettent pas de les rattacher à une des deux espèces. Au total, les résultats RAPD permettent d'assigner un nom d'espèce à 11 des 18 souches préalablement non identifiées.

L'analyse RAPD a permis de mettre en évidence des similarités entre souches différentes et de constituer des groupes. C'est ainsi que 26 des 30 souches de $L b$ plantarum sont regroupées dans G4. Toutes les souches de Lb pentosus, quant à elles, sont regroupées dans G1, G2 ou G3. Comme nous avons utilisé deux amorces différentes pour classer nos souches, il est possible d'examiner l'effet du choix de l'amorce sur le classement final obtenu. La comparaison des classements effectués avec l'amorce B06 ou B08 ou les deux, indique que les résultats obtenus ne sont pas entièrement comparables. Si les deux amorces permettent de détecter les souches identiques ou très similaires, elles ne permettent pas, utilisées seules, de regrouper aussi bien les souches moins similaires. C'est ainsi que la sonde B06 forme trois groupes de Lb plantarum que l'utilisation supplémentaire de la sonde B08 permet de rassembler au sein du seul groupe G4. Ce résultat s'explique par une caractéristique de l'analyse RAPD : la classification est d'autant meilleure qu'un plus grand nombre de bandes est analysé.

\section{DISCUSSION ET PERSPECTIVES}

L’analyse de la diversité des souches de $L b$ plantarum et de $L b$ pentosus de la collection du CNRZ par RAPD a permis de beaucoup clarifier le statut de ces souches. Nous avons montré que près de $40 \%$ des souches étaient des doublons ou des souches très semblables qui pouvaient être soustraites de la collection sans affecter sa représentativité. De plus, cette analyse a permis de mettre en évidence les différentes souches d'une même espèce et isolées d'un même type de produits (fermentations d'olives vertes d'Espagne, par exemple). Ces résultats suggèrent l'intérêt de l'analyse par RAPD pour ceux qui cherchent à caractériser les flores spontanées sauvages. Il est ainsi possible de faire l'inventaire des différentes souches de la même espèce présentes dans un même habitat, tout en évitant de collecter et d'étudier des souches en nombre inutilement élevé.

Nous avons aussi assigné un nom d'espèce à $61 \%$ des souches dont la situation taxonomique était incertaine. Cela a été rendu possible par le regroupement des souches à l'aide de la RAPD associée à un calcul informatisé des coefficients de similarité entre profils, à une analyse des données par la méthode UPGMA et à la constitution d'une banque de données de profils RAPD. Ces résultats soulignent les limites des techniques classiques d'identification et l'intérêt des techniques de typage moléculaire en taxonomie bactérienne.

Cependant, toutes les souches n'ont pu être reliées valablement les unes aux autres dans notre étude. L'identification de certaines d'entre elles demeure problématique. II est possible qu'en utilisant d'autres amorces, des similarités puissent être détectées entre groupes actuellement distincts. 
II est également possible que certaines souches, bien que présentant le phénotype typique d'une espèce, n'y appartiennent pas. Le problème se pose particulièrement pour les souches des groupes G1 à G3. Si G3, qui contient la souche type, est réellement un groupe de $L b$ pentosus, il peut ne pas en être de même pour les souches de G1 ou de G2. Pour lever ce doute, il est nécessaire d'utiliser d'autres techniques telles que la détermination d'homologie ADN/ADN ou de séquence d'ARN $16 S$. D'ores et déjà, des mesures d'homologie $A D N / A D N$ ont établi que le groupe G1 est effectivement constitué de $L b$ pentosus (Bringel et al, communication personnelle).

En conclusion, la RAPD apparaît comme une puissante méthode d'identification et de typage des micro-organismes. Elle a d'importantes applications potentielles, non seulement pour la constitution et l'enrichissement dirigés des collections mais également pour la microbiologie fondamentale. Dans la mesure où elle révèle la diversité existant au sein des espèces, l'analyse par RAPD des populations permet d'identifier la ou les quelques souches représentant au mieux l'espèce et celles correspondant à des individus rarement rencontrés. Elle conduit donc à un choix plus rationnel des souches modèles à étudier de façon plus approfondie. Et, combinée à d'autres techniques, elle devrait faciliter l'identification de nouvelles espèces bactériennes.

\section{REMERCIEMENTS}

Nous remercions vivement nos collègues qui ont eu la gentillesse de nous confier leurs souches et notamment M El Soda (Dept of Dairy Technology, Faculty of Agriculture. Alexandria University, Alexandrie, Egypte) ; R Jiménez-Diaz (Instituto de la Grasa y sus Derivados, Dept Biotecnologia de Alimentos, Seville, Espagne); S Tanasupawat (Chulalongkorn University, Bangkok, Thailande) et L Topisirovic (Institute of Molecular Genetics and Genetic Engineering, Belgrade, Yougoslavie).

\section{RÉFÉRENCES}

Brikun I, Suziedelis K, Berg DE (1994) DNA sequence divergence among derivatives of Escherichia coli $\mathrm{K}$ 12 detected by arbitrary primer PCR (Random amplified polymorphic DNA) fingerprinting. J Bacteriol 176, 1673-1682

Dellaglio F, Bottazzi V, Vescovo M (1975) Deoxyribonucleic acid homology among Lactobacillus species of the subgenus Streptobacterium Orla-Jensen. Int $J$ Syst Bacteriol 25, 160-172

de los Reyes-Gavilan CG, Limsowtin GKY, Tailliez P, Séchaud L, Accolas JP (1992) A Lactobacillus helveticus-specific DNA probe detects restriction fragment length polymorphisms in this species. Appl Environ Microbiol 58, 3429-3432

Fekete A, Bantle JA, Halling SM, Stich RW (1992) Amplification fragment length polymorphism in Brucella strains by use of polymerase chain reaction with arbitrary primers. J Bacteriol 174, 7778-7783

Kersulyte D, Woods JP, Keath EJ, Goldman WE, Berg DE (1992) Diversity among clinical isolates of Histoplasma capsulatum detected by polymerase chain reaction with arbitrary primers. J Bacteriol 174, 70757079

Le Bourgeois P, Lautier M, Ritzenthaler P (1993) Chromosome mapping in lactic acid bacteria. FEMS Microbiol Rev 12, 109-124

Sneath PHA, Sokal RR (1973) Numerical taxonomy: The principles and practice of numerical classification. WH Freeman, San Francisco

Sokal RR, Michener CD (1958) A statistical method for evaluating systematic relationships. Univ Kansas Sci Bull 38, 1409-1438

Vauterin L, Vauterin P (1992) Computer-aided objective comparison of electrophoresis patterns for grouping and identification of microorganisms. Eur Microbiol 1, 37-41

Welsh J, McClelland M (1990) Fingerprinting genomes using PCR with arbitrary primers. Nucleic Acids Res $18,7213-7218$

Welsh J, Pretzman C, Postic D, Saint Girons I, Baranton G, McClelland M (1992) Genomic fingerprinting by arbitrarily primed polymerase chain reaction resolves Borrelia burgdorferi into three distinct phyletic groups. Int $J$ Syst Bacteriol 42, 370-377

Williams JGK, Kubelick AR, Livak KJ, Rafalski JA, Tingey SV (1990) DNA polymorphisms amplified by arbitrary primers are useful as genetic markers. Nucleic Acids Res 18, 6531-6535

Zanoni P, Farrow JAE, Phillips BA, Collins MD (1987) Lactobacillus pentosus (Fred, Peterson, and Anderson) sp nov, nom rev. Int $J$ Syst Bacteriol 37, 339341 